\title{
Problem Solving AlgortihM For THE MANUfactruring DePARTMENT USINg AgENT TECHNOLOGY
}

\author{
POPA, C.L.; PARPALA, L.F. \& AURITE, T.
}

Abstract: A cooperative system represents a system where different users or agents work together at the same project from different locations. Agent technology has been recognized as an important approach for developing distributed design and manufacturing systems. In this paper we will present an algorithm that agents can use in order to solve a problem occurred in a department of an enterprise which is part of a cooperative system. Also we will present an example of information exchange between agents, using a simplified communication system.

Key words: agent technology, distributed system, algorithm, influence agent
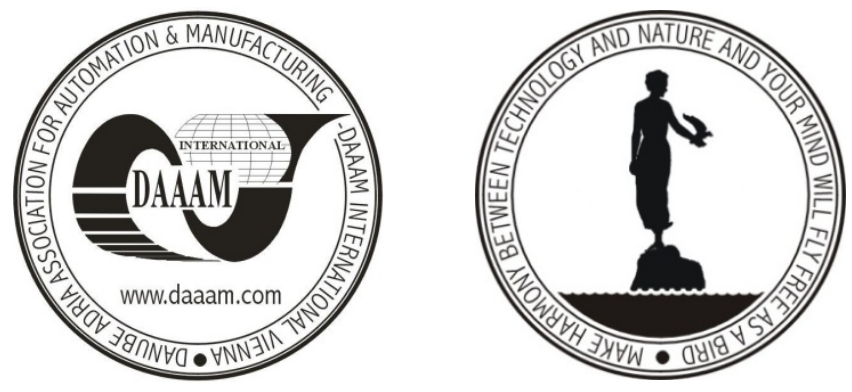

Authors' data: Dr. Ass. Prof. Ph.D. Popa, C[icerone] L[aurentiu]; Dr. Ass. Prof. PhD. Parpala, L[idia] F[lorentina]; Prof. Dr. Aurite, T[raian], University Politehnica of Bucharest, IMST Faculty, MSP department, UPB - PREMINV research center, 313, Spl. Independentei, 060042, Bucharest, RO, laurentiubest@yahoo.com, lidia.parpala@gmail.com, aurite@amcsit.ro

This Publication has to be referred as: Popa, C[icerone] L[aurentiu]; Parpala, L[idia] F[lorentina] \& Aurite, T[raian] (2008). Problem Solving Algortihm for the Manufactruring Department Using Agent Technology, Chapter 51 in DAAAM International Scientific Book 2008, pp. 603-612, B. Katalinic (Ed.), Published by DAAAM International, ISBN 978-3-901509-66-7, ISSN 1726-9687, Vienna, Austria DOI: $10.2507 /$ daaam.scibook.2008.51 